\title{
Note on Continued Fractions and the Sequence of Natural Numbers
}

\author{
By H. W. Turnboll.
}

When a student first approaches the theory of infinite continued fractions a natural question that suggests itself is how to evaluate the expression

$$
f=\frac{1}{2+}-\frac{3}{4+} \frac{5}{6+} \ldots \frac{2 n-1}{2 n+} \ldots \text { : }
$$

but, as far as I know, it is considered in no text book short of the great work by O. Perron, Die Lehre von dem Kettenbruchen (Teubner, 1913). I propose to consider this, and also an allied fraction

$$
g=1+\frac{2}{3+} \frac{4}{5+} \frac{6}{7+} \cdots \frac{2 n}{(2 n+1)+\cdots}
$$

in an elementary fashion, and incidentally to point out the curious contrast in their properties which seems to reside in this simple displacement of the ascending sequence of natural numbers. For example, in $f$, the first fraction, the integers are arranged in a zigzag sequence, which gives rise to the second fraction by moving each element one place along the zigzag in a general leftwards direction-a retreating step, let us say. Evidently the repetition of such a step restores the general features of $f$ (when one considers the general term); and the same remark applies to an even number of such steps. In this way one is led to consider the more general fraction

$$
h=\frac{1}{a+1+} \frac{a+2}{a+3+} \frac{a+3}{a+4+} \cdots,
$$

which is equal to $f$ when $a=1$, and to $g^{-1}$ when $a=0$. Even values of $a$ correspond to $g$ and to fractions derived from $g$ by an even number of retreating steps, whereas odd values of $a$ correspond to $f$ and all such even derivatives of $f$.

Evidently there are two classes-the odd, or $f$ class, and the even, or $g$ class-of these fractions, which at first sight appear to differ hardly at all in complexity. A little further examination will shew that while each member of the $g$ class can be evaluated rationally in terms of the elementary transcendental number $\sqrt{\bar{e}}$, no such simple result follows for the $f$ class. 
If, instead of starting with the sequence $1,2,3, \ldots$, one duplicates it (taking a kind of second power of the sequence) by writing $1,1,2,2,3,3, \ldots$, and then proceeds to form a zigzag of odd or even order, the result is at once related to $\sqrt{ } e \cdot \sqrt{ } e=e$. For example:

$$
\begin{aligned}
e & =2+\frac{2}{2+} \frac{3}{3+} \frac{4}{4+} \ldots=2 \cdot 7182818284 \ldots \\
& =2+\frac{1}{1+} \frac{1}{2+} \frac{2}{3+} \frac{3}{4+} \ldots
\end{aligned}
$$

Here it matters not whether an odd or an even number of retreating steps is taken; all such continued fractions are evidently of the form. $(\alpha+\beta e) /(\gamma+\delta e)$, where $\alpha, \beta, \gamma, \delta$ are positive integers. For instance, from the first of this pair of continued fractions we may deduce

$$
\frac{3}{3+} \frac{4}{4+} \ldots=\frac{2}{e-2}-2=\frac{6-2 e}{e-2} .
$$

Fractions of this type (4) which may be found in most text books on algebra, are collected by Perron upon p. 209 of the cited work, among a number of other interesting expressions for $e$ and $e^{x}$. They originate in formulae known to Euler, Cesàro, and Lagrange.

In passing it may be suggested that further interesting results would probably follow by placing the third and higher powers of the integer sequence upon a zigzag, in the manner explained; (or again the sequence of prime numbers might be attempted!). Ultimately such an enquiry includes the case $1,1,1,1, \ldots$, which leads to the Fibonacci numbers $1,2,3,5,8, \ldots$ by way of the recurring continued fraction $1+.1+\frac{1}{1+} \cdots$

To evaluate $f$, let $p_{n} / q_{n}$ be the $n^{\text {th }}$ convergent; so that, in the usual way, we have

$$
\left.\begin{array}{ll}
p_{1}=1, p_{2}=4, & p_{n}=2 n p_{n-1}+(2 n-1) p_{n-2}, \\
q_{1}=2, q_{2}=11, & q_{n}=2 n q_{n-1}+(2 n-1) q_{n-2 .} .
\end{array}\right\}
$$

By using this difference relation upon each term of the series

$$
p_{n}-p_{n-1}+p_{n-2}-\ldots+(-)^{n} p_{2},
$$

and also upon the analogous series for $q$, we obtain

$$
\left.\begin{array}{l}
p_{n}=(2 n+1) p_{n-1}-(-1)^{n}, \\
q_{n}=(2 n+1) q_{n-1}+(-1)^{n} .
\end{array}\right\}
$$


By addition, $p_{n}+q_{n}=(2 n+1)\left(p_{n-1}+q_{n-1}\right)$, which leads by repetition to the relation

$$
p_{n}+q_{n}=1.3 .5 \ldots(2 n-1)(2 n+1) .
$$

On using the repeated relations (6), after dividing throughout by $2 n+1$, we obtain

$$
\frac{q_{n}}{q_{n}+p_{n}}=1-\frac{1}{3}+\frac{1}{3.5}-\frac{1}{3.5 .7}+\ldots+(-)^{n} \frac{1}{3.5 \ldots(2 n+1)},
$$

and also

$$
f=\lim _{n \rightarrow \infty} \frac{p_{n}}{q_{n}}=\frac{\frac{1}{3}-\frac{1}{3.5}+\frac{1}{3.5 .7}-\ldots}{1-\frac{1}{3}+\frac{1}{3.5}-\frac{1}{3.5 .7}+\ldots}=\cdot 37973 \ldots
$$

(There is a strange reluctance for even digits to appear in the work).

Exactly the same procedure avails for the more general continued fraction $h$, which includes $g$. Thus in the same notation for the convergents of $h$, so that

$$
\begin{array}{ll}
p_{1}=1 \quad, & p_{n}=(a+2 n-1) p_{n-1}+(a+2 n-2) p_{n-2}, \\
q_{1}=a+1, & q_{n}=(a+2 n-1) q_{n-1}+(a+2 n-2) q_{n-2},
\end{array}
$$

\section{Hence}

$$
\begin{aligned}
& p_{n}=(a+2 n) p_{n-1}-(-)^{n} 1, \\
& q_{n}=(a+2 n) q_{n-1}+(-)^{n} 1 .
\end{aligned}
$$

also

$$
p_{n}+q_{n}=(a+2)(a+4) \ldots(a+2 n) ;
$$

$\frac{q_{n}}{p_{n}+q_{n}}=1-\frac{1}{a+2}+\frac{1}{(a+2)(a+4)}-\frac{1}{(a+2)(a+4)(a+6)}+\ldots$ to $n+1$ terms.

If $a=0$ and if $n \rightarrow \infty$, this last series is at once identified with

$$
1-\frac{1}{2}+\frac{1}{2.4}-\frac{1}{2.6}+\ldots=e^{-\frac{1}{2}}=\cdot 6065 \ldots
$$

Thus

$$
\frac{1}{1+\lim _{n \rightarrow \infty} p_{n} / q_{n}}=e^{-\frac{1}{1}}
$$

so that the continued fraction $g$ has been evaluated as

$$
\frac{1}{e^{\frac{3}{3}}-1}=1+\frac{2}{3+} \frac{4}{5+} \frac{6}{7+} \cdots
$$


xii

Hence $g$ and, as already explained, any continued fraction obtained by an even number of retreating steps from $g$, may be expressed as a bilinear form $(a \sqrt{ } e+\beta) /(\gamma \sqrt{ } e+8)$ with integer coefficients. For example, by unwrapping this last relation sufficiently far, we obtain

$$
\frac{6}{7+} \frac{8}{9+} \ldots=-5+\frac{4}{-3+} \frac{2}{-1+} \frac{1}{\sqrt{ } e-1}=\frac{48-29 \sqrt{ } e}{5 \sqrt{ } e-8}
$$

This consideration also shews that the above series (11) can be summed in terms of $\sqrt{ } e$ for all even positive integral values of $a$, as also is at once apparent on dividing the series throughout by the product 2.4.....a.

On the contrary, no such convenient result follows for odd integral values of $a$, which lead to the $f$ class already discussed.

A series of the type to which (11) belongs, in which the numerators are all equal while the denominators are accumulating products which retain all preceding factors, are sometimes looked upon as ascending continued fractions in contrast to the usual descending kind. By means of an obvious notation, whose use is illustrated by the example

$$
\frac{\alpha+}{\beta} \frac{\gamma}{\delta}=\frac{\alpha+\frac{\gamma}{\delta}}{\beta}
$$

the relation (11) may be thrown into the form

$\frac{1}{1+h}=\frac{1}{1+} \frac{1}{(a+1)+} \frac{a+2}{(a+3)+} \cdots=1-\frac{1-}{a+2} \frac{1-}{a+4} \frac{1-}{a+6} \ldots$

for all values of $a$ except negative even integral values.

Once again, each term of this same series (11) may be resolved into partial fractions and re-sorted into groups such as $\frac{1}{a+2 r} \Sigma_{r}$, where $\Sigma_{r}$ is an absolutely convergent series independent of $a$, when $n \rightarrow \infty$. Thus

$$
\frac{q_{n}}{p_{n}+q_{n}}=1+\frac{\Sigma_{1}}{a+2}+\frac{\Sigma_{2}}{a+4}+\frac{\Sigma_{3}}{a+6}+\ldots+\frac{\Sigma_{n}}{a+2 n},
$$

where the value of $\Sigma_{r}$ may be found, in the usual way, by multiplying throughout by $a+2 r$ and then putting $a=-2 r$ in the result. This yields the expression whose limit, when $n \rightarrow \infty$, is

$$
\frac{1}{1+h}=1-e^{-\frac{1}{2}}\left(\frac{1}{a+2}+\frac{1}{2(a+4)}+\frac{1}{2.4(a+6)}+\ldots\right) \text {. }
$$


This holds for all values of $a$ which do not cause the denominators to vanish. Here again it follows that any series of this last type can be summed as a bilinear form in $\sqrt{ } e$ if $a$ is an even positive integer.

The essential distinction between the $f$ and the $g$ classes can now be exhibited. Let

$$
y=\frac{x^{a+2}}{a+2}+\frac{x^{a+4}}{2(a+4)}+\frac{x^{a+6}}{2.4} \frac{(a+6)}{(a+\ldots}
$$

a series which converges for all finite values of $x$, and admits of a derivative

$$
\frac{d y}{d x}=X^{a+1}\left(1+\frac{x^{2}}{2}+\frac{x^{4}}{2 \cdot 4}+\ldots\right)=x^{a+1} \exp \left(\frac{x^{2}}{2}\right) .
$$

It follows, by integration for the range $0 \leqslant x \leqslant 1$, that

$$
\frac{1}{1+h}=1-e^{-\frac{1}{2}} \int_{0}^{1} x^{a+1} e^{x^{2} / 2} d x
$$

which can evidently be evaluated very simply by parts, if, and only if, $a$ is zero or an even positive integer. But for all other values of $a$, including the odd, or $f$, class, this integral is by no means easy to track. It has some affinity with the function

$$
P(\mu)=\int_{0}^{1} u^{\mu-1} e^{-u} d u
$$

of Saalschütz (cf. Whittaker and Watson, Modern Analysis (1915) 238 ), where $2 \mu=a+1$, but its range of integration extends from 0 to $-\frac{1}{2}$ and not from 0 to 1 .

In conclusion it may be mentioned that the method employed above to derive the relations (6) equally well applies to establish the results (4). It then exhibits the same features as would be found in the recurrence relation whereby the number of permutations of $n$ letters $a_{1}, a_{2}, \ldots, a_{n}$ may be enumerated in the case when each letter is displaced from its original position, in each permutation.

\section{Note on Polynomial Interpolation}

By A. C. Aitren.

The formulae of interpolation of Lagrange and Newton are easily retained in the memory if one considers a simple way in which each can be derived, for polynomials. 